\title{
Search for electric dipole moments in storage rings
}

\author{
Paolo Lenisa ${ }^{1, \star}$ \\ ${ }^{1}$ University of Ferrara and INFN, Ferrara, Italy
}

\begin{abstract}
The JEDI collaboration aims at making use of storage ring to provide the most precise measurement of the electric dipole moments of hadrons. The method makes exploits a longitudinal polarized beam. The existence an electric dipole moment would generate a torque slowly twisting the particle spin out of plan of the storage ring into the vertical direction. The observation of non zero electric dipole moment would represent a clear sign of new physics beyond the Standard Model. Feasiblity tests are presently undergoing at the COSY storage ring Forschungszentrum Jülich (Germany), to develop the novel techniques to be implemented in a future dedicated storage ring.
\end{abstract}

\section{Introduction}

The fact that we and the world around us are made of matter and only a minimal amount of antimatter is observed, constitutes one of the fundamental puzzles in modern physics, motivating a variety of theoretical speculations and experimental investigations. The combined standard models of cosmology and particle physics suggest that at the end of the inflation epoch immediately following the Big Bang, the number of particles and antiparticles were almost in precise balance. Yet the laws of physics contrived to act differently on matter and antimatter to generate the apparently large imbalance that we observe today. One of the necessary mechanisms required for this to happen - namely CP violation - is very small in the Standard Model of particle physics and therefore only able to account for a tiny fraction of the actual imbalance. New sources of $\mathrm{CP}$ violation are needed, and one such potential signature would be the appearance of electric dipole moments (EDMs) in fundamental particles.

An EDM originates from a permanent charge separation inside the particle. In its centre-of-mass frame, the ground state of a subatomic particle has no direction at its disposal except its spin, which is an axial vector, while the charge separation (EDM) corresponds to a polar vector (see Fig. 1). Therefore, if such a particle with nonzero mass and spin possesses an EDM, it must violate both parity $(\mathrm{P})$ and time-reversal $(\mathrm{T})$ invariance. If the combined CPT symmetry is to be valid, $\mathrm{T}$ violation also implies breaking of the combined CP symmetry. The Standard Model predicts the existence of EDMs, but their sizes (in the range of $10^{-31}$ to $10^{-33} \mathrm{e} \cdot \mathrm{cm}$ for nucleons) fall many orders of magnitude below the sensitivity of current measurements and still far below the expected levels of projected experiments. An EDM observation at a much higher value would therefore be a clear and convincing sign of new physics beyond the current Standard Model (BSM).

BSM theories such as supersymmetry (SUSY), technicolour, multi-Higgs models and left-right symmetric models generally predict nucleon EDMs in the range of $10^{-24}$ to $10^{-28} \mathrm{e} \cdot \mathrm{cm}$ (part of the

^e-mail: lenisa@fe.infn.it 
upper region of this range is already excluded by experiment). Although tiny, EDMs of this size would be large enough to be observed by a new generation of highly sensitive accelerator based experiments with charged particles such as the proton and deuteron. In this respect, EDMs offer a complementary approach to searches for BSM physics at collider experiments, probing scales far beyond the reach of present high-energy machines such as the LHC. For example, in certain SUSY scenarios the present observed EDM limits provide information about physics at the $\mathrm{TeV}$ or even $\mathrm{PeV}$ scales, depending on the mass scale of the supersymmetric mechanisms and the strength of the CP-violating SUSY phase parameters (Fig.2).

Researchers have been searching for EDMs in neutral particles, especially neutrons, for more than 50 years, by trapping and cooling particles in small volumes and using strong electric fields. Despite an enormous improvement in sensitivity, however, these experiments have only produced upper bounds. The current upper limit of approximately $10^{-26} \mathrm{e} \cdot \mathrm{cm}$ for the EDM of the neutron is an amazingly accurate result: if we had inflated the neutron so that it had the radius of the Earth, the EDM would correspond to a separation between positive and negative charges of about $1 \mathrm{~mm}$. An upper limit of less than $10^{-29} \mathrm{e} \cdot \mathrm{cm}$ has also been reported for a special isotope of mercury, but the Coulomb screening by number to the permanent EDMs of the neutrons and protons in its nucleus. For the electron, meanwhile, the reported EDM limits on more complicated polar molecules can be used to deduce a bound of about $10^{-28} \mathrm{e} \cdot \mathrm{cm}$ - which is even further away from the Standard Model prediction $\left(10^{-38} \mathrm{e} \cdot \mathrm{cm}\right)$ than is the case for the neutron.

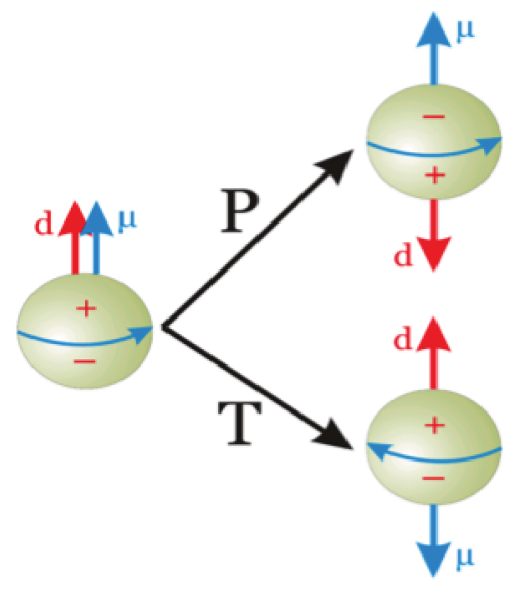

Figure 1. Naively, an electric dipole moment (d) and a magnetic dipole moment $(\mu)$ transfer differently under $\mathrm{P}$ and $\mathrm{T}$. In a fundamental particle, both quantities are proportional to the spin vector (s). Therefore, the interaction term (ds. E) is odd under P and T, whereas $(\mu \mathrm{s} \cdot \mathrm{B})$ is even under these transformations.

\section{Perspectives for storage rings}

Although these experiments provide useful constraints on BSM theories, a new class of experiments based on storage rings is needed to measure the electric dipole moment of charged particles (such as the proton, deuteron or helium-3). These highly sensitive acceleratorbased experiments will allow the EDM of charged particles to be inferred from their very slow spin precession in the presence of large electric fields, and promise to reach a sensitivity of $10^{-29} \mathrm{e} \cdot \mathrm{cm}$. This is due mainly to the larger number of particles available in a stored beam compared with the ultra-cold neutrons usually found in trap experiments, and also the potentially longer observation time possible because such experiments are not limited by the particle decay time. Storage-ring experiments would span the range of EDM sizes where new CP violation is expected to lie. Furthermore, the ability to measure EDMs of more 


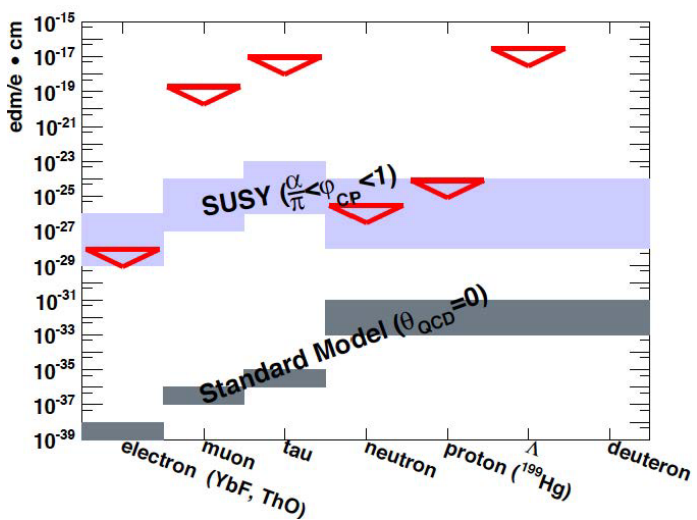

Figure 2. Experimental upper limits for the EDMs of different particles (red arrows) plotted together with the prediction from SUSY (blue bands) and the Standard Model (grey). No experimental limit exists yet for the deuteron.

than one type of particle will help to constrain the origin of the CP-violating source because not all particles are equally sensitive to the various CP-violating mechanisms.

At the Cooler Synchrotron "COSY" located at the Forschungszentrum Jülich (FZJ), Germany (Fig.3), the JEDI (Jülich Electric Dipole moment Investigations) Collaboration ${ }^{1}$ is working on a series of feasibility studies for such a measurement using an existing conventional hadron storage ring. COSY, which is able to store both polarised proton and deuteron beams with a momentum up to 3.7 $\mathrm{GeV} / \mathrm{c}$, is an ideal machine for the development and commissioning of the necessary technology. This $\mathrm{R} \& \mathrm{D}$ work has recently replaced COSY's previous hadron-physics programme of particle production and rare decays, although some other service and user activities continue.

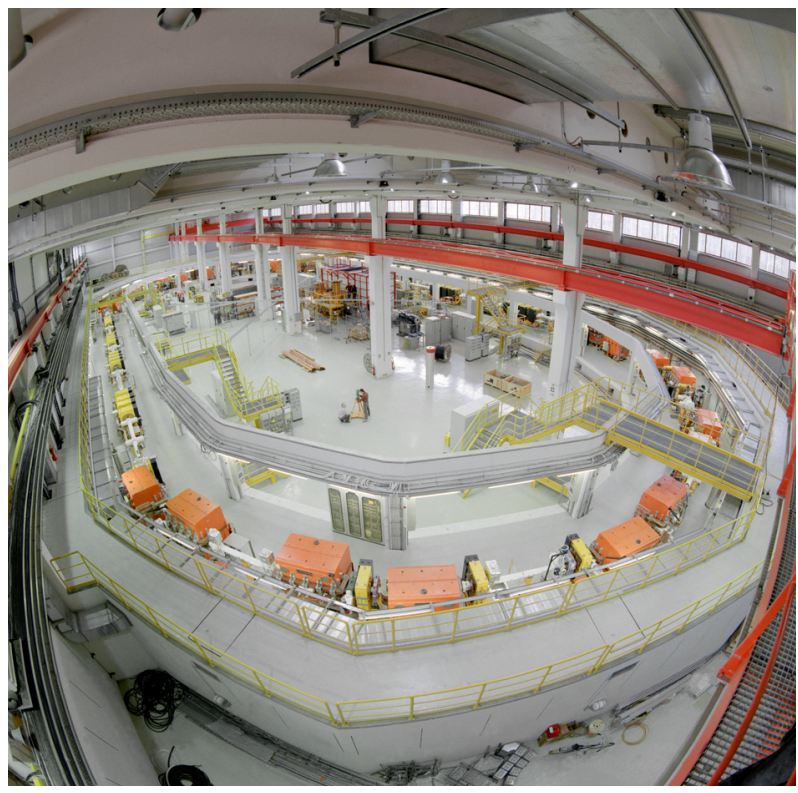

Figure 3. The COSY storage ring at the Forschungszentrum Jülich in Germany.

\footnotetext{
${ }^{1}$ http://collaborations.fz-juelich.de/ikp/jedi/index.shtml
} 
A first upper limit for an EDM directly measured in a storage ring was obtained for muons at the (g-2) experiment at Brookhaven National Laboratory (BNL) in the US, but the measurement was not optimised for sensitivity to the EDM. Subsequently, scientists at BNL began to explore what would be needed to fully exploit the potential of a storage-ring experiment. While much initial discussion for an EDM experiment also took place at Brookhaven, commitments to the Relativistic Heavy Ion Collider (RHIC) operation and planning for a potential Electron-Ion Collider have prevented further development of such a project there. Therefore the focus shifted to FZJ and the COSY storage ring in Germany, where the JEDI collaboration was formed in 2011 to address the EDM opportunity.

The measuring principle is straightforward: a radial electric field is applied to an ensemble of particles circulating in a storage ring with their polarisation vector (or spin) initially aligned with their momentum direction. Maintaining the polarisation in this direction requires a storage ring in which the bending elements are a carefully matched set of both vertical magnetic fields and radial electric fields. The field strengths must be chosen such that the precession rate of the polarisation matches the circulation rate of the beam (called the "frozen spin"). For particles such as the proton with a positive gyromagnetic anomaly, this can be achieved by using only electric fields and choosing just the right "magic" momentum value (around $0.7 \mathrm{GeV} / \mathrm{c}$ ). For deuterons, which have a negative gyromagnetic anomaly, a combination of electric and magnetic fields is required, but in this case the frozen spin condition can be achieved for a wide range of momentum and electric/magnetic field combinations. Such combined fields may also be used for the proton and would allow the experiment to operate at momenta other than the magic value.

The existence of an EDM would generate a torque that slowly rotates the spin out of the plane of the storage ring and into the vertical plane (see panel opposite). This slow change in the vertical polarisation is measured by sampling the beam with elastic scattering off a carbon target and looking for a slowly increasing left-right asymmetry in the scattered particle flux. For an EDM of $10^{-29}$ e.cm and an electric field of $10 \mathrm{MV} / \mathrm{m}$, this would happen at an angular velocity of $3 \cdot 10^{-9} \mathrm{rad} \cdot \mathrm{s}^{-1}$ (about 1/100th of a degree per day of continuous operations). This requires the measurement to be sensitive at a level never reached before in a storage ring. To obtain a statistically significant result, the polarisation in the ring plane must last for approximately $1000 \mathrm{~s}$ during a single fill of the ring, while the scattering asymmetry from the carbon target must reach levels above $10^{-6}$ to be measurable within a year of running.

\section{Achievements at COSY}

Following the commissioning of a measurement system that stores the clock time of each recorded event in the beam polarimeter with respect to the start of the accelerator cycle, the JEDI collaboration has passed a series of important milestones in recent years. Working with the deuteron beam at COSY, these time stamps make it possible to unfold for the first time the rapid rotation of the polarisation in the ring plan (which has a frequency of around $120 \mathrm{kHz}$ ) that arises from the gyromagnetic anomaly. In a one-second time interval, the number of polarisation revolutions may be counted and the final direction of the polarisation known to better than 0.1 rad (Fig. 4)[1]. The magnitude of the polarisation may decline slowly due to decoherence effects in storage ring, as can be seen in subsequent polarisation measurements within a single fill.

Maintaining the polarisation requires the cancellation of effects that may cause the particles in the beam to differ from one another. Bunching and electron cooling serves to remove much of this spurious motion, but particle path lengths around the ring may differ if particles in the beam have transverse oscillations with different amplitudes. Recently, we demonstrated that the effect of these differences on polarisation decoherence can be removed by applying correcting sextupole fields to the 
ring. As a result, we can now achieve polarisation lifetimes in the horizontal plane of more than 1000 $\mathrm{s}-$ as required for the EDM experiment (Fig. 5) [2]. In the past year, the JEDI group has also shown that by determining errors in the polarisation direction and feeding this back to make small changes in the ring's radio-frequency, the direction of the polarisation may be maintained at the level of 0.1 rad during any chosen time period. This is a further requirement for managing the polarisation in the ring for the EDM measurement.

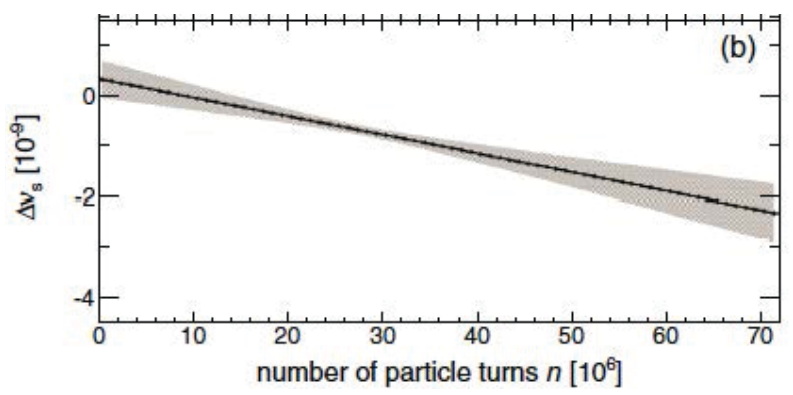

Figure 4. Deviation of the spin tune $v_{s}$, which is defined as the number of spin precessions per turn, as a function of the number of turns in the ring. At $\mathrm{t}=38 \mathrm{~s}$ (about $28 \times 10^{6}$ turns), the interpolated spin tune amounts to $16097540628.3 \pm 9.7 \times 10^{-11}$, which represents the most precise measurement of this quantity ever performed. The previous best measurement, performed for the muon at the (g-2) experiment, had a precision of $3 \times 10^{-8}$ per year. The higher precision achieved here is mainly attributed to the much longer measurement time of $100 \mathrm{~s}$ compared with $600 \mu$ in the (g-2) experiment.

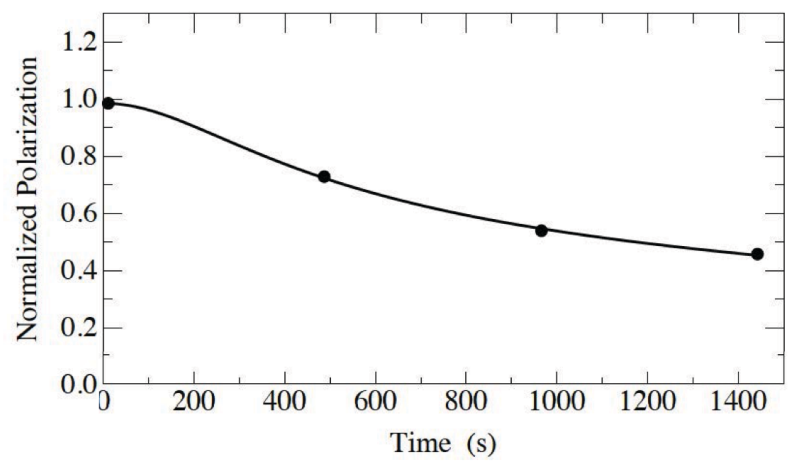

Figure 5. One of the longest polarisation lifetimes recorded for the COSY ring. Measurements made at four separate times (to conserve beam) are matched to a depolarisation curve that assumes a Gaussian distribution of transverse oscillation amplitudes. The half-life of the polarisation is $1173 \pm 172 \mathrm{~s}$, which is three orders of magnitude longer than previous results using electron beams.

COSY is also an important test facility for many EDM-related technologies, among them new beam-position monitoring, control and feedback systems. High electric fields and combined electric/ magnetic deflectors may also find applications in other fields, such as accelerator science. Many checks for systematic errors will be undertaken, and a technical design report for a future dedicated storage ring will be prepared. The most significant challenges will come from small imperfections 
in the placement and orientation of ring elements, which may cause stray field components that generate the accumulation of an EDM-like signal. The experiment is most sensitive to radial magnetic fields and vertical electric fields. Similar effects may arise through the non-commutativity of spurious rotations within the ring system, and efforts are under way to model these effects via spin tracking supported with beam testing. Eventually, many such effects may be reduced or eliminated by comparing the signal accumulation rates seen with beams travelling in opposite directions in the storage ring. During the next decade, this will allow researchers to approach the design goals of the EDM search using a storage ring, adding a new opportunity to unveil physics beyond the Standard Model.

\section{ERC-Advanced grant}

In early 2016, the European Research Council awarded an advanced research grant to the Juelich group to support further developmental efforts. The five-year grant, starting in October, will support a consortium that also includes RWTH Aachen University in Germany and the University of Ferrara in Italy. The goal of the project is to conduct the first measurement of the deuteron EDM. Since the COSY polarisation cannot be maintained parallel to its velocity (because no combined electric and magnetic bending elements exist), a novel device called a radiofrequency Wien filter will be installed in the ring to slowly accumulate the EDM signal (the filter influences the spin motion without acting on the particle's orbit) [3]. The idea is to exploit the electric fields created in the particle rest system by the magnetic fields of the storage-ring dipoles, which would allow the first ever measurement of the deuteron EDM.

\section{References}

[1] D. Eversmann et al., Phys. Rev. Lett. 115, 094801 (2015)

[2] G. Guidoboni et al., Phys. Rev. Lett. 116, 054801 (2016)

[3] J. Slim et al., Nucl. Instr. and Meth. A 828, 116 (2016) 